\title{
Trends in Bacteria Causing Diarrheal Infection from 2010 to 2018 in Cheonan, Korea: Aeromonas spp., Campylobacter spp., and Clostridioides spp.
}

\author{
Ji On Park and Jae Kyung Kim* \\ Department of Biomedical Laboratory Science, Dankook University College of Health Sciences, Cheonan 31116, Republic of Korea
}

Received: March 5, 2019 / Revised: April 17, 2019 / Accepted: April 19, 2019

\begin{abstract}
Diarrhea is one of the most common infectious diseases known worldwide. However, few studies have examined anaerobic diarrhea-causing bacteria (DB), which are difficult to culture. Recent advances in molecular biology have facilitated the detection and analysis of anaerobic DB. In this study, long-term trends in anaerobic DB were evaluated in Korea. From 2010 to 2018, symptoms of diarrhea reported were analyzed among patients hospitalized at the Dankook University Hospital in Korea. Results of multiplex polymerase chain reaction based on seasonality, age, overlapping infection, and other factors in patients were evaluated. DB were detected in $38.2 \%$ of 1716 stool specimens in the duration of the study. Of the pathogens detected using this method, $49.8 \%(\mathrm{n}=405 / 813)$ were anaerobic bacteria, including Clostridioides perfringens, Campylobacter spp., Clostridioides difficile toxin B, and Aeromonas spp. Among the four anaerobic bacteria, Clostridioides perfringens was the most commonly occurring $(15.5 \% ; n=126 / 813)$. Detection rates of Clostridioides perfringens, Clostridioides difficile toxin B, and Aeromonas spp. were $34.1 \%(\mathrm{n}=22 /$ $55), 34.9 \%(n=43 / 126)$, and $40.0 \%(n=38 / 109)$, respectively. The detection rate of Campylobacter spp. $(32.7 \%$; $n=37 / 115)$ was the highest in patients between 10 and 20 years of age. The detection rate of anaerobic DB showed an increase in 2018 as compared with that in 2010, and the number of events of diarrhea caused by anaerobic DB also increased in this duration. Further studies are required to devise methods that might prevent the proliferation of anaerobic DB.
\end{abstract}

Keywords: Multiplex polymerase chain reaction, anaerobic bacteria, diarrhea-causing bacteria

\section{Introduction}

Gastroenteritis with diarrhea is a common gastrointestinal disorder that affects more than 3-5 billion individuals worldwide each year [1] and is caused by various pathogens, including bacteria, viruses, and protozoa [2]. Patients with gastroenteritis with diarrhea may develop dehydration symptoms due to diarrhea, leading to death if proper treatment is not obtained.

\section{*Corresponding author}

Tel: +82-41-550-1451, Fax: +82-41-550-1451

E-mail: nerowolf2@dankook.ac.kr

๑ 2019, The Korean Society for Microbiology and Biotechnology
Research on diarrhea-causing bacteria (DB) has been carried out in various fields for many years. However, few studies have evaluated the distribution and epidemiological trends in anaerobic DB for which culture is difficult. Recent molecular biology experiments have facilitated the detection of anaerobic DB that are difficult to culture, enabling the analysis of trends in anaerobic DB over a relatively long period of time.

Clostridioides perfringens is a gram-positive anaerobic bacterium that produces spores and is found in the gastrointestinal tracts of humans and animals [3]. Spores of Clostridioides perfringens are not killed after heating for $4 \mathrm{~h}$ at $100^{\circ} \mathrm{C}$ and can, therefore, persist when food is kept 
at a warm temperature for an extended period of time, such as the conditions encountered in cafeterias and hospitals [4]. In addition to simple gastroenteritis with diarrhea, Clostridioides perfringens can also cause enteritis necroticans [5].

Campylobacter spp. is a gram-negative anaerobic strain that is mainly distributed in the intestines of companion animals [6]. Campylobacter spp. is transmitted through undercooked meat, water contaminated with livestock feces, raw milk, and pasteurized milk products. In recent years, the detection rate of Campylobacter spp. in the United States of America has gradually increased [7].

Owing to the public health implications of gastroenteritis with diarrhea, national surveillance of this disease has been conducted in several countries, including EnterNet-Korea in Korea, EnterNet in the European Union, FoodNet in the United States of America, OzFoodNet in Australia, and FoodNet Canada in Canada. Despite national research and surveillance of DB, trend analysis studies focusing on anaerobic bacteria are rare.

Accordingly, in this study, the current status and trends of isolation of DB were evaluated in order to contribute to basic data on the treatment and prevention of gastroenteritis with diarrhea.

\section{Materials and Methods}

This study was approved by Dankook University Research Ethics Committee (IRB No. 2018-06-007).

\section{Samples}

From June 2010 to May 2018, patients with diarrhea who were admitted to Dankook University Hospital were examined. Stool specimens were evaluated using multiplex polymerase chain reaction (mPCR). The samples were subjected to nucleic acid extraction within $24 \mathrm{~h}$ after collection.

\section{Nucleic acid extraction}

For DNA isolation for mPCR, a QIAamp DNA Mini Kit (Qiagen, Germany) was used according to the manufacturer's instructions. For each sample, $200 \mu \mathrm{l}$ sample was used for DNA isolation. A Nanodrop 1000 (Thermo
Table 1. Target genes and their product sizes for multiplex PCR.

\begin{tabular}{lcc}
\hline \multicolumn{1}{c}{ Pathogen } & Target genes & Product size (bp) \\
\hline Aeromonas spp. & hly, ela & 217 \\
Campylobacter spp. & asp, hip & 227 \\
Clostridioides perfringens & $c p a$ & 700 \\
Clostridioides difficile toxin B & $t c d B$ & 476 \\
Escherichia coli H7 & fliC & 370 \\
Escherichia coli O157 & rfb & 476 \\
Salmonella spp. & sopB & 395 \\
Shigella spp. & vif, ipaH & 330 \\
Vibrio spp. & hly, tlh, vvh & 651 \\
Verocytotoxin-producing & VT1, vT2 & 291 \\
Escherichia coli & & \\
Yersinia enterocolitica & inv & 580
\end{tabular}

Fisher Scientific, USA) was used to measure the concentration of extracted DNA.

\section{mPCR analysis}

For PCR, a Seeplex Diarrhea-B1 and B2 ACE Detection kit (Seegene, Korea) was used. Ten pathogen species, including Aeromonas spp., Campylobacter spp., Clostridioides difficile toxin B, Clostridioides perfringens, Escherichia coli 0157:H7, Salmonella spp., Shigella spp., verocytotoxin-producing $E$. coli, Vibrio spp., and Yersinia enterocolitica, were evaluated. Four microliters of 5× DB1 (DB2) PM (containing $1 \mu$ l of 8-methoxypsoralen solution and $3 \mu \mathrm{l}$ primer mixture containing primer pairs for the internal control and template for the internal control) in $2 \times$ Multiplex Master Mix (containing DNA polymerase, buffer with deoxynucleoside triphosphates, $\mathrm{MgCl}_{2}$, and stabilizers) was added. Next, $17 \mu \mathrm{l}$ PCR Master Mix and $3 \mu \mathrm{l}$ nucleic acid were added to PCR tubes, and PCR was performed. PCR target genes and product sizes are shown in Table 1.

\section{Electrophoresis}

The mPCR products were electrophoresed on $2 \%$ agarose gels with ethidium bromide for $30 \mathrm{~min}$ at $100-$ $150 \mathrm{~V}$. The agarose gels were rinsed with distilled water, amplification was assessed by visualizing the gel on a UV trans illuminator, and the results were analyzed after photographs were acquired. 


\section{Results}

Among 1,716 stool specimens, DB were detected in 656 samples (average detection rate: $38.2 \%$ over the 9 year period). Among these 656 samples, 813 pathogens were detected. The four anaerobic DB accounted for $49.8 \%$ ( $n=405 / 813$ ) of these pathogens. Of 1,716 patients, 919 were male, and 797 were female. The detection rate of DB in males was $38.6 \%(n=355 / 919)$, whereas that in females was $37.8 \%(\mathrm{n}=301 / 797)$. The average age of the patients was 36.5 years (median age, 29.8 years; range, 0.0-97.7 years), and the average age of patients with DB positivity was 32.5 years (median age, 19.0 years; range, 0.0-97.7 years).

The detection ratio of Clostridioides perfringens was the highest in November $(14.3 \%$; $\mathrm{n}=18 / 126)$, and detection ratio of Campylobacter spp. was the highest in July $(19.1 \% ; \mathrm{n}=22 / 115)$. The detection ratio of Clostridioides difficile toxin B was highest in May $(12.8 \% ; n=14 / 109)$, and detection ratio of Aeromonas spp. was highest in August (20.0\%; $\mathrm{n}=11 / 55$; Table 2$)$.

As shown in Fig. 1, the PCR-positive detection rate for anaerobic DB was the highest at 45.1\% $(n=101 / 224)$, whereas that of aerobic bacteria was $43.6 \%(n=41 / 94)$. The anaerobic DB detection rate was the highest (65.5\%; $\mathrm{n}=36 / 55)$ in patients 20-30 years of age, whereas the aerobic DB detection rate was the highest $(62.8 \% ; \mathrm{n}=$

Table 2. Monthly distribution of diarrhea-causing bacteria detection rates from 2010 to 2018 in Cheonan, Korea.

\begin{tabular}{|c|c|c|c|c|c|}
\hline \multicolumn{2}{|c|}{ Month } & \multirow{2}{*}{$\begin{array}{c}\text { Clostridioides } \\
\text { perfringens } \\
(\%)\end{array}$} & \multirow{2}{*}{$\begin{array}{c}\text { Campylo- } \\
\text { bacter spp. } \\
(\%)\end{array}$} & \multirow{2}{*}{$\begin{array}{c}\text { Clostridioides } \\
\text { difficile toxin } \\
\text { B (\%) } \\
7.3\end{array}$} & \multirow{2}{*}{$\begin{array}{c}\text { eromonas } \\
\text { spp. } \\
(\%)\end{array}$} \\
\hline Spring & 3 & & & & \\
\hline & 4 & 6.3 & 1.7 & 11.0 & 0.0 \\
\hline & 5 & 11.1 & 12.2 & 12.8 & 9.1 \\
\hline \multirow[t]{3}{*}{ Summer } & 6 & 9.5 & 13.9 & 9.2 & 5.5 \\
\hline & 7 & 10.3 & 19.1 & 9.2 & 14.5 \\
\hline & 8 & 8.7 & 18.3 & 5.5 & 20.0 \\
\hline \multirow[t]{3}{*}{ Autumn } & 9 & 7.1 & 9.6 & 8.3 & 16.4 \\
\hline & 10 & 6.3 & 2.6 & 9.2 & 16.4 \\
\hline & 11 & 14.3 & 7.0 & 6.4 & 7.3 \\
\hline \multirow[t]{3}{*}{ Winter } & 12 & 4.0 & 6.1 & 6.4 & 1.8 \\
\hline & 1 & 4.8 & 1.7 & 5.5 & 1.8 \\
\hline & 2 & 4.8 & 2.6 & 9.2 & 3.6 \\
\hline
\end{tabular}

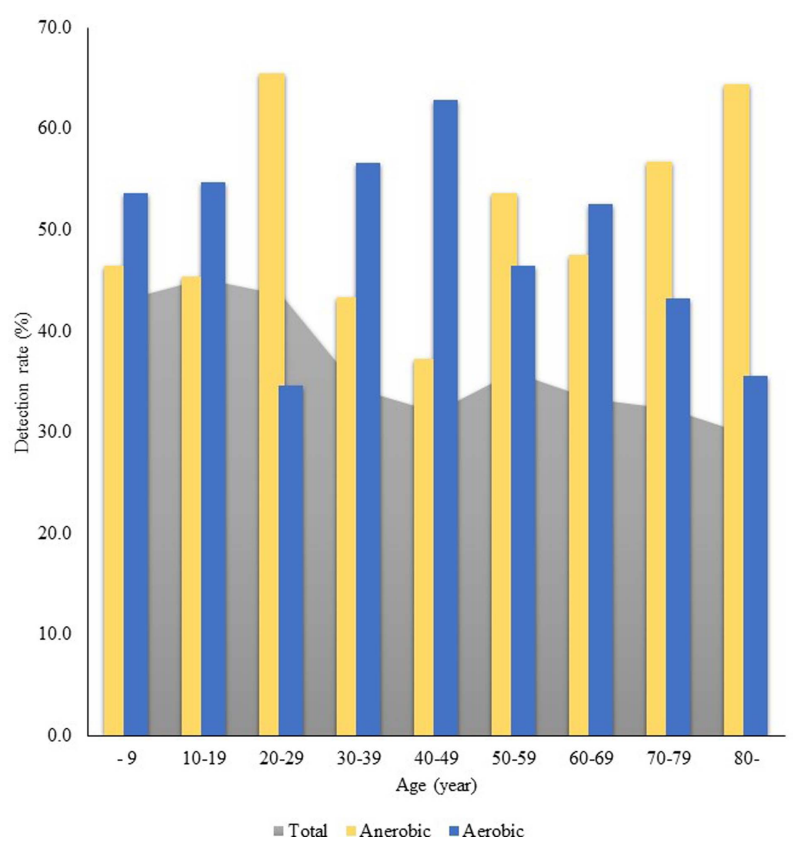

Fig. 1. Age distribution of diarrhea-causing bacteria detection rates (2010-2018) in Cheonan, Korea.

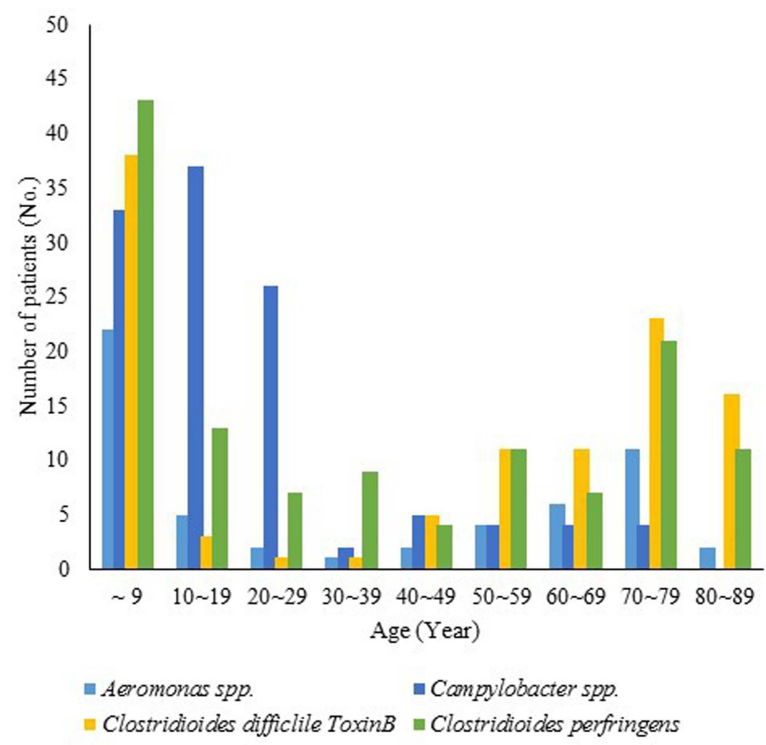

\begin{tabular}{|c|c|}
\hline Pathogen & Average age \\
\hline Aeromonas spp. & 34.8 \\
\hline Camphlyobacter spp. & 21.3 \\
\hline Clostridioides difficlile ToxinB & 44.9 \\
\hline Clostridioides perfringens & 36.0 \\
\hline
\end{tabular}

Fig. 2. Age distribution by pathogen and Average age of patients that tested positive for anaerobic diarrhea-causing bacteria. 
$27 / 43$ ) in patients $40-49$ years of age.

Detection rates of Clostridioides perfringens, Clostridioides difficile toxin B, and Aeromonas spp. (Fig. 2) were higher in patients less than 10 years of age and greater than 70 years of age, and the detection rate of Campylobacter spp. was the highest in patients 10-20 years of age.

The average age of patients with Campylobacter spp. positivity was the lowest (21.3 years; median age: 16.1 years; range: 0.0-77.8 years), and that of patients with Clostridioides difficile toxin B positivity was 44.9 years (median age: 58.0 years; range: $0.5-93.0$ years; Fig. 2).

The co-infection rate for DB detected by PCR was $20.1 \%(\mathrm{n}=132 / 656)$. Of these individuals, 26 coinfections involved anaerobic DB, accounting for 19.7\% of DB co-infections. Of co-infections between the anaerobic DBs, 24 were double infections, and two were triple infections. Among co-infections with anaerobic DB, 10 cases of double infections of Clostridioides perfringens and Campylobacter spp. were observed.

As shown in Fig. 3, the detection rates of Clostridioides perfringens were $10.8 \%(n=4 / 37)$ in 2010 and $32 \%(n=$ $8 / 25)$ in 2018. The detection rate of Campylobacter spp. increased from $10.8 \%(\mathrm{n}=4 / 37)$ in 2010 to $28.0 \%(\mathrm{n}=7 /$ 25) in 2018, and the detection rate of Clostridioides difficile toxin B also increased from $2.7 \%$ in 2010 to $24.0 \%$ in

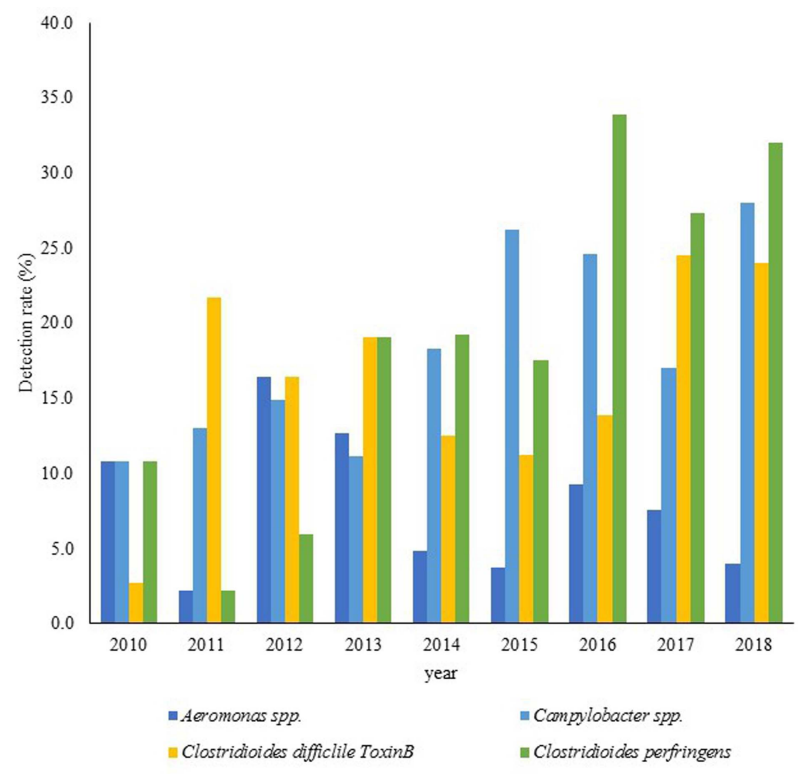

Fig. 3. Distribution of diarrhea-causing bacteria detection rates (2010-2018) in Cheonan, Korea.

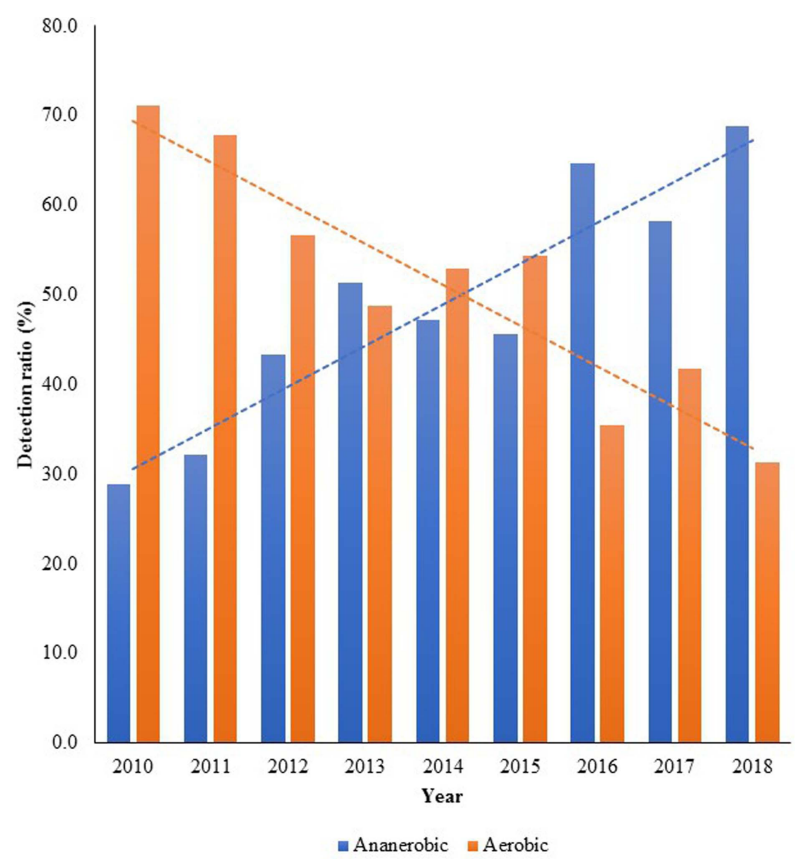

Fig. 4. Distribution of anaerobic and aerobic diarrhea-causing bacteria detection ratios (2010-2018) in Cheonan, Korea.

$2018(\mathrm{n}=6 / 25)$. The detection rate of anaerobic DB increased from $28.9 \%$ in $2010(\mathrm{n}=13 / 45)$ to $68.8 \%$ in $2018(n=22 / 32)$.

\section{Discussion}

In this study, the incidence and characteristics of DB positivity in Korean patients hospitalized for diarrhea at Dankook University Hospital from 2010 to 2018 were examined. The results of this study showed that the DBpositive rate during the study period was higher than those reported by Korea Centers for Disease Control and Prevention (KCDC) in 2013 [8], Kim et al. in 2013 [9], and Lee et al. in 2018 [10]. Among anaerobic DB, Clostridioides perfringens was the most common. The Acute Diarrheal Disease Laboratory Surveillance Project in Korea reported that the detection rate of Clostridioides spp. was high in Chungnam province [9]. In this study, Clostridioides perfringens was detected not only in anaerobic DB but also among $10 \mathrm{DB}$ samples analyzed by PCR. The next most commonly detected anaerobic DB was Campylobacter spp., which was identified at a higher rate in this study than in a study by the KCDC in 2013 [8]. According to surveillance data (C-EnterNet) published in Canada [11], Campylobacter spp. was rec- 
ognized as the first pathogen of food poisoning bacteria, and the World Health Organization (WHO) identified Campylobacter spp. as the most common causative pathogen of gastroenteritis with diarrhea [12]. Therefore, active prevention of Campylobacter spp. is essential.

The detection rate increased from May and was highest in August in this study. This trend was similar to that described by Kim et al. [9]. Moreover, the detection rate of DB decreased between December and January and tended to increase between June and August, similar to the results of a study described by Lee et al. [10] in 2018. Campylobacter spp. tended to be highly detected throughout the year, mainly from May to August, and Clostridioides perfringens was detected at high levels in March and November, similar to the results described by the KCDC [8] and Kim et al. [9] in 2013. Aeromonas spp. showed the highest detection rates from August to October. Bacteria are generally known to grow vigorously in hot and humid environments, resulting in higher detection and disease rates during the summer months. However, the results showed that Aeromonas spp. was evenly distributed throughout the year, not only in June and August. Thus, measures for DB prevention are needed throughout the year.

The detection rate of Clostridioides difficile toxin B was the highest in patients less than 10 years of age and was decreased in patients 10-40 years of age. These results were similar to those of another Korean study [10,13]. Clostridioides perfringens, Clostridioides difficile toxin B, and Aeromonas spp. showed the highest detection rates in patients less than 10 years of age. The average age of anaerobic DB-positive patients was the lowest in patients infected with Campylobacter spp. Indeed, Campylobacter spp. is the most common in children less than 1 year of age and in individuals aged between 15 and 44 years; the reason for this is still unknown [14].

Few studies have examined co-infection rates and frequencies of anaerobic DB. In general, standardized laboratory methods for diagnosing DB infection involve confirming the presence of the pathogen after incubation $[15,16]$. In this study, co-infection frequently occurred between anaerobic DBs, with Clostridioides perfringens and Campylobacter spp. being the most common, followed by Clostridioides perfringens and Clostridioides difficile toxin B. Moreover, two triple infections between anaerobic DBs (one case of triple infection with Clos- tridioides perfringens, Clostridioides difficile toxin B, and Aeromonas spp. and one case of triple infection with Clostridioides difficile toxin B, Campylobacter spp., and Aeromonas spp.) were observed. Duplicate infections among DBs are common in developing countries, and little is known about the biological interactions between infected strains [17, 18]. Thus, further studies are required to evaluate the clinical relevance of overlapping infections.

Detection rates of Clostridioides perfringens, Campylobacter spp., and Clostridioides difficile toxin B increased in 2018 compared with those in 2010. Compared with previous studies in Korea, the detection rate of Campylobacter spp. has increased steadily [9, 19]. The presence of Clostridioides difficile toxin B has increased with the use of antibiotics [20]. Indeed, in this study, the rate in 2018 was almost 10 times higher than that in 2010. Aeromonas spp. also has high intestinal colonization rates in Korea because of the high intake of raw fish or uncooked seafood [21]. However, the mechanism through which anaerobic DB cause increased diarrhea is not clear. More research is needed to evaluate this effect of anaerobic DB in Korea.

There were some limitations to this study. First, this study was conducted at a single institution and did not represent various regions throughout Korea. Moreover, this was a retrospective study, and the results were not analyzed according to the clinical characteristics of the patients (symptoms, previous antibiotic administration, underlying diseases, etc.). In addition, antibiotic resistance could not be studied in this context. Only DB infection trends from 2010 to 2018 were analyzed, and further studies are needed to assess other pathogens (e.g., viruses) causing diarrhea in the future.

Most diarrhea symptoms are temporary and do not require treatment or laboratory findings for confirmation of the causative pathogen. However, because severe health problems can result in young patients or patients with underlying diseases, it is necessary to promptly and accurately distinguish and diagnose the causative bacteria. In particular, the possibility of recurrence of Campylobacter spp. is increasing because of treatment difficulties [22]. Some recent studies have examined the prevalence of anaerobic DB in diarrheal diseases. Therefore, the current findings are expected to be helpful for the treatment and prevention of diarrheal diseases 
caused by anaerobic bacteria. A common method for diagnosing bacterial infection in diarrhea is to identify pathogens after culture $[15,16]$. However, because anaerobic DB are not easily cultured, it is difficult to identify anaerobic bacteria using this method. In contrast, PCR is useful for diagnosing anaerobic DB with high accuracy. Thus, further studies are being planned to develop a new DB rapid detection kit that identifies anaerobic DB species in cases in which diarrheal disease does not spontaneously resolve.

\section{Conflict of Interest}

The authors have no financial conflicts of interest to declare.

\section{References}

1. Foodborne Disease Burden Epidemiology Reference Group. WHO estimates of the global burden of foodborne diseases. World Health Organization, 2015.

2. Lee S, Park Y, Lee HK, Kim S, Kim J, Lee S. et al. 2013. Detection of 13 enteric bacteria and 5 viruses causing acute infectious diarrhea using multiplex PCR from direct stool specimens. Ann. Clin. Microbiol. 16: 33-38.

3. Ryu C, Lee M. 2011. Food poisoning. J. Korean Med. Assoc. 54: 617-626.

4. Centers for Disease Control. Clostridioides perfringens. 2017. Available from: https://www.cdc.gov/foodsafety/diseases/Clostridioides-perfringens.html. Accessed 21 Aug. 2018.

5. Clarke LE, Diekmann Guiroy B, Mcnamee W, Java DJ Jr, Weiss SM. 1994. Enteritis necroticans with midgut necrosis caused by Clostridioides perfringens. Arch. Surg. 129: 557-560.

6. CDC. Campylobacter [Internet]. CDC. Available from: https:// www.cdc.gov/campylobacter/. Accessed 15 April 2019.

7. Geissler AL, Bustos Carrillo F, Swanson K, Patrick ME, Fullerton KE, Bennett $C$, et al. 2017. Increasing Campylobacter infections, outbreaks, and antimicrobial resistance in the United States, 20042012. Clin. Infect. Dis. 65: 1624-1631.

8. KCDC. The Prevalence and Characteristics of Bacteria causing Acute Diarrhea in Korea, 2013. Public Health Weekly Report KCDC. 2013 8: 70-74. Available from: www.cdc.go.kr. Accessed 27 Dec. 2018.

9. Kim N, Cha I, Kim J, Chung GT, Kang Y, Hong S. 2013. The preva- lence and characteristics of bacteria causing acute diarrhea in Korea. Kor. J. Clin. Microbiol. 16: 174-181.

10. Lee K, Kim DS, Chong MS. 2018. Multiplex PCR based epidemiological study for the causes of acute diarrheal disease in adults living in Jeju Island. Ann. Clin. Microbiol. 21: 1-7.

11. Government of Canada. C-EnterNet. 2018. Available from: https:/ /www.canada.ca/en/public-health/services/surveillance/foodnet-canada.html. Accessed 10 Jan. 2019.

12. World Health Organization. Campylobacter. 2018. Available from: https://www.who.int/news-room/fact-sheets/detail/campylobacter. Accessed 15 Jan. 2019.

13. Lee J, Kim J, Cho H, Oh K. 2015. Detection of bacterial and viral pathogens in stool specimens using multiplex PCR. J. Lab. Med. Qual. Assur. 37: 141-147.

14. Acheson D, Allos BM. 2001. Campylobacter jejuni infections: update on emerging issues and trends. Clin. Infect. Dis. 32: 12011206.

15. Fan H, Wu Q, Kou X. 2008. Co-detection of five species of waterborne bacteria by multiplex PCR. Life Sci. J. 5: 47-54.

16. Khamrin P, Okame M, Thongprachum A, Nantachit N, Nishimura $S$, Okitsu $S$, et al. 2011. A single-tube multiplex PCR for rapid detection in feces of 10 viruses causing diarrhea. J. Virol. Methods 173: 390-393.

17. Lindsay B, Ramamurthy $T$, Gupta $S$, Takeda $Y$, Rajendran K, Nair $\mathrm{GB}$, et al. 2011. Diarrheagenic pathogens in polymicrobial infections. Emerg. Infect. Dis. 17: 606-611.

18. Bhavnani D, Goldstick JE, Cevallos W, Trueba G, Eisenberg JN. 2012. Synergistic effects between rotavirus and coinfecting pathogens on diarrheal disease: evidence from a communitybased study in northwestern Ecuador. Am. J. Epidemiol. 176: 387395.

19. Cho SH, Shin HH, Choi YH, Park MS, Lee BK. 2008. Enteric bacteria isolated from acute diarrheal patients in the Republic of Korea between the year 2004 and 2006. J. Microbiol. 46: 325-330.

20. Chitnis AS, Holzbauer SM, Belflower RM, Winston LG, Bamberg WM, Lyons C, et al. 2013. Epidemiology of community-associated Clostridioides difficile infection, 2009 through 2011. JAMA Intern. Med. 173: 1359-1367.

21. Oh YH, Kim YB. 1988. Use of polymerase chain reaction and serum antibodies for diagnosis of enterohemorrhagic Escherichia coli. J. Korean Soc. Microbiol. 33: 99-110.

22. Randremanana RV, Randrianirina F, Sabatier P, Rakotonirina HC, Randriamanantena A, Razanajatovo IM, et al. 2014. Campylobacter infection in a cohort of rural children in Moramanga, Madagascar. BMC Infect. Dis. 14: 372. 